\title{
Integrated force and distance sensing using elastomer-embedded commodity proximity sensors
}

\author{
Radhen Patel and Nikolaus Correll \\ Department of Computer Science, University of Colorado, Boulder, CO 80309, USA
}

\begin{abstract}
We describe a combined force and distance sensor using a commodity infrared distance sensor embedded in a transparent elastomer with applications in robotic manipulation. Prior to contact, the sensor works as a distance sensor $(0-10 \mathrm{~cm})$, whereas after contact the material doubles as a spring, with force proportional to the compression of the elastomer ( 0 $5 \mathrm{~N}$ ). We describe its principle of operation and design parameters, including polymer thickness, mixing ratio, and emitter current, and show that the sensor response has an inflection point at contact that is independent of an object's surface properties. We then demonstrate how two arrays of eight sensors, each mounted on a standard Baxter gripper, can be used to (1) improve gripper alignment during grasping, (2) determine contact points with objects, and (3) obtain crude 3D models that can serve to determine possible grasp locations.
\end{abstract}

\section{INTRODUCTION}

Grasping and manipulation remain hard challenges in robotics. After identifying an object's pose, the robot's end-effector needs to be controlled to create a sufficient number of constraints for successful pick-up while maintaining the object's pose until all desired contact points are reached, thereby preventing the object from moving out of the end-effector's reach. The ability to use in-hand sensing to better align with an object and stop exerting forces once contact is made, might allow to provide these constraints without affecting the object's pose. In [15], touch sensors are used to determine whether a grasp is successful, but cannot be used to improve the grasp prior to contact. Exploiting environmental constraints such as walls [11] or a bowl [14 has been shown to increase grasping success in specific scenarios, but planning such a motion requires precise knowledge of the environment's geometry and would benefit from active sensing to determine whether an object has reached a desired pose. Using 3D sensing suffers from uncertainty, making reliable grasps very difficult, regardless of the type of end-effector used.

Yet, commercially successful systems, that is widely deployed, in-hand sensors are virtually non-existing as they are difficult to manufacture and expensive. At the same time, the algorithmic foundations for reactive grasp planning are only sparsely developed, with most of the focus on the sense-plan-act model that requires precise sensing and actuation. This has been nicely illustrated at the 2015 Amazon Picking Challenge, where only one of 25 teams have used tactile feedback for contact sensing [10].

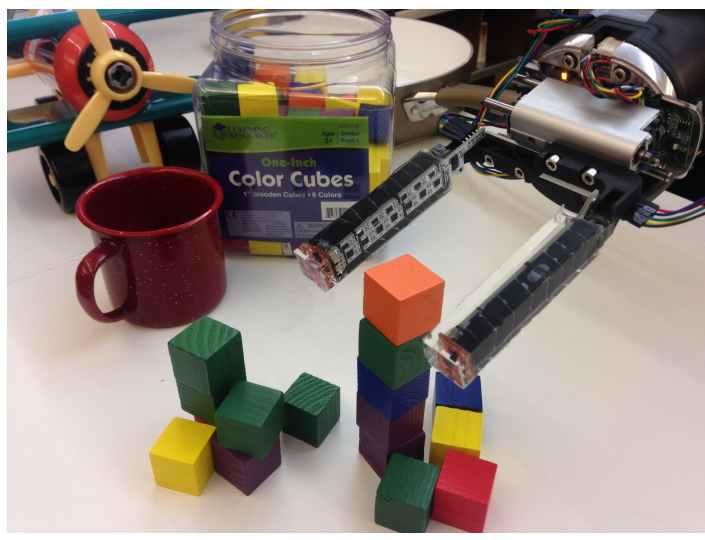

Fig. 1. A Baxter parallel gripper enhanced by two arrays of combined force and proximity sensors. Seven sensors are spaced along each finger's contact area, and one sensor is mounted at each tip, enabling object detection and force control.

Motivated by this apparent gap both in available hardware and applications for in-hand sensing, we present a simple and low-cost tactile sensor that combines force and distance measurements. The proposed sensor is simple to manufacture and easy to integrate with existing hardware. The sensor consists of a commodity digital infrared distance sensor that is embedded in a soft polymer, which doubles as a spring for force measurements based on Hooke's law. We also show how the strong dependence of infrared-based sensors on surface properties can be overcome by exploiting the discontinuity that the elastomer coating introduces into the sensor response. After describing other sensors that are closest to the work presented here in Sec. I-A we explain the sensor's manufacturing and principle of operation in Sec. II The impact of various design parameters is shown in Sec. III and calibration results are shown in Sec. IV] We then shown how the proposed sensor can be arranged into an array and installed on a parallel gripper of Rethink Robotic's Baxter to improve grasping performance during selective tasks in Sec. V

\section{A. Related work}

Tactile sensing is widely considered an essential capability for efficient grasping and manipulation [12. By touching an object, it is possible to measure contact properties such as contact forces, contact position, and possibly even gather information about an object's surface properties. At the same time, sensors need to be rugged enough 
to withstand chemical and mechanical abrasion. Various types of tactile sensors have been investigated based on strain gauges, piezoelectric, capacitive, impedance, mechanical and magnetic effects 12. For example, a silicon piezoresistive sensor measures both compressive and shear forces at the skin-object interface in 36. A capacitive tactile sensor reached a resolution of $0.05 \mathrm{~N}$ over the range of $10 \mathrm{~N}$ for forces in both normal and tangential directions in 38. A soft tactile sensor, comprising multiple capacitors embedded in polydimethylsiloxane (PDMS), was reported with a minimum detectable force of $10 \mathrm{mN}$ in [27. Fiber Bragg gratings based sensors enable small size and high sensitivity. A soft fiber optic sensor based on polymer fiber Bragg gratings is embedded into a soft PDMS film in [37, for simultaneous measurement of shear and normal stresses. A fiber optical sensor for a multifingered robotic hand was designed in [23] for contact force and contact location sensing. Galinstan-based strain sensors have been embedded into soft robotic fingers to measure curvature and - in conjunction with pressure detect the presence of objects [16] and adjust a grasp if necessary [17. Finally, simple microphones can be used to measure vibrations that allow identifying textures $[22$. How to use all of this tactile information during control is a topic of ongoing research [13, 4].

Part of the challenges with manufacturing and signal processing that all of the above sensors share to some extent, can be countered by using commodity digital sensors. Barometric pressure sensors covered with flexible polymers can provide contact and pressure sensing [35. Similarly, optical proximity sensors have been integrated into deformable rubber domes 24 and urethane foam 20, 34, 18, which both alter light reflection upon compression and shear, a phenomenon known as frustrated reflection. Finally, [26] combines off-the-shelf proximity sensors embedded in elastomers with capacitive sensing to perform directional force sensing.

In addition to contact/force, equipping robotic hands [2. 21] or skin [32] with distance sensors is attractive to improve a grasp during the approach phase. Optical proximity sensors were integrated inside the fingertips of a Barrett Hand in order to perform reactive online grasping [21. In 29], the fingertips of robot manipulator TUMRosie were equipped with proximity sensors to measure the distance to objects.

There also exist approaches that use both distance and force simultaneously. In [19], capacitive sensors are used to measure both proximity of (conductive) objects as well as pressure after contact. In 31, optical proximity and capacitive pressure sensing are combined to realize a multimodal tactile sensing skin. In this paper, we demonstrate how both distance and force sensing can be achieved using a single off-the-shelf sensor, allowing for making the device small enough for finger-tip operation, simple to manufacture, and low-cost, while providing performance that is comparable to the above approaches.

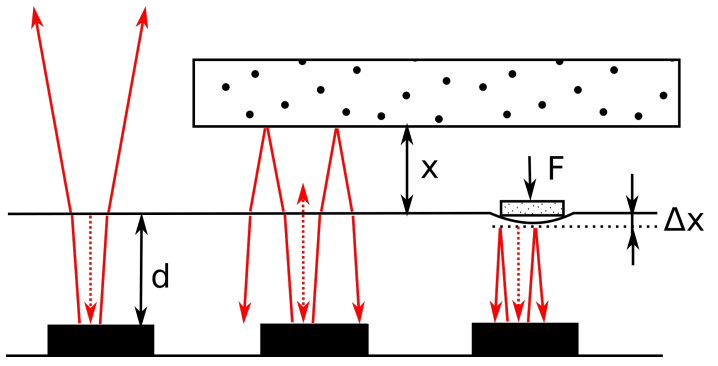

Fig. 2. Schematic sensor design illustrating key quantities. Infrared lobes are reflected at the interface of PDMS/air due to Fresnel reflection, as well as from close-by objects. Forces lead to deformation of the PDMS that reduces its width $d$ by $\Delta x$.

\section{SEnsor DESIGN}

Infrared sensors are strongly non-linear and their response depends on the surface properties of the sensed objects and the angle of incidence. They are sensitive to cross-talk from other sensors or infrared light in the environment. Increasing use in consumer electronics such as smart phones has led to a new generation of devices that improve cross-sensitivity by integrating sensor and emitter with digital signal processing.

We have chosen an integrated proximity and ambient light sensor VCNL 4010 (Vishay Semiconductors). This device has a miniature $3.95 \times 3.95 \times 0.75 \mathrm{~mm}^{3}$ package which combines an infrared emitter and PIN photodiode for proximity measurement, ambient light sensor, a signal processing IC, a 16 bit ADC, and inter-integrated-circuit $\left(I^{2} C\right)$ communication interface while requiring only very few external components (four filter capacitors). The chip allows setting a large variety of parameters, the most important being the emitter current $(20 \mathrm{~mA}$ to $200 \mathrm{~mA}$ in increments of $10 \mathrm{~mA}$ ), and the carrier frequency in the range from $390.625 \mathrm{kHz}-3.125 \mathrm{MHz}$ in four increments. The ability to select the frequency of each sensor enables arranging sensors in opposite pairs, such as required on a robotic gripper, without interference. The emitter current should not be confused with the actual power consumption, which is less than $4 \mathrm{~mA}$ when performing 250 measurements per second at full $(200 \mathrm{~mA})$ power, and in the order of $\mu \mathrm{A}$ when doing 10 or less measurements per second.

Sensors can be arranged in groups of 8 using an $I^{2} C$ multiplexer (TCA9548A, Texas Instrument). This chip has a programmable 3-bit address, allowing us to create arrays of up to $8 \times 8$ sensors. At $100 \mathrm{kHz} I^{2} C$ bus frequency, a single measurement requires $1470 \mu \mathrm{s}$ including communication, allowing to read a $8 \times 8$ array at $10 \mathrm{~Hz}$ and a strip of eight at $85 \mathrm{~Hz}$.

To enable force measurements, sensors are embedded in a thin layer of PDMS (Dow Corning Sylgard 184), see also Figure 2 PDMS is a widely used silicon elastomer, whose mechanical and optical properties have been widely studied [6, 7, 9]. It is simple to manufacture and cheap, while providing good transparency and mechanical properties such as resistance to chemical and mechanical abrasion. 
Figure 1 shows two sensor arrays mounted to the parallel gripper of a "Baxter" robot.

\section{A. Principle of operation}

The integrated infrared emitter of the VCNL4010 has a peak wavelength of $890 \mathrm{~nm}$. The light from the emitter passes through a thin layer of PDMS. It is then reflected by nearby objects and received by the photo-receiver. The amplitude and phase of the light vary as a function of the distance to the surface, its orientation, color and texture.

Due to the quadratic decay of light amplitude with distance, the sensor has its highest resolution right after its minimum range of $0.5 \mathrm{~mm}$. It is therefore possible to measure small variations in the order of hundredths of millimeters. We exploit this effect by measuring the elastic deformation that occurs when an object is pressed against the sensor. As the elastomer can be approximated by a spring with a constant Young's modulus $E$ (for light pressure), the force is given by

$$
F \approx \frac{E A}{d} \Delta x
$$

with $A$ the contact area over the sensor, $d$ the width of the PDMS layer and $\Delta x$ the measured deformation. Note that the sensor area is constant and smaller than the actual contact area of typical objects. Yet, the value of $F$ is approximate as PDMS cannot be infinitely compressed and eventually changes its density and thereby absorption properties.

Let the emitted light intensity be $I_{0}$ and the measured reflected intensity from an object be $I$. Let the thickness of the rubber be $d$ and the distance to the object $x$, see also Figure 2. Depending on the index of refraction of the rubber material, a fraction $R$ of the light will be reflected from the interface between rubber and air, a fraction $\kappa$ will be scattered, and a fraction $\alpha$ will be reflected at the target surface. Assuming that the light intensity decays quadratically with distance, we can approximate the amount of returned infrared as

$$
I_{x>0} \approx I_{0}(1-R) \frac{\alpha}{(d+x)^{2}}+I_{0} R \frac{1}{d^{2}}-\kappa I_{0} .
$$

The reflection at the PDMS/air interface can be calculated using the Fresnel equation [5], which reduces to

$$
R=\left|\frac{n_{1}-n_{2}}{n_{1}+n_{2}}\right|^{2}
$$

for normal incidence. With the refractive index of PDMS $n_{1} \approx 1.41$ and that of air $n_{2} \approx 1$, around $2.9 \%$ of the light gets reflected from the internal surface of the PDMS as well as on the outside on the return path.

This formalism helps us to better understand certain edge cases. First, when $d \ll x$, the light intensity at the receiver is dominated by $\frac{I_{0} R}{d^{2}}$, which leads to saturation of the sensor, if $I_{0}$ is too large or $d$ is too small. The width $d$ of the PDMS therefore governs the maximum current at which we can operate the sensor and thereby the maximum attainable range. At the same time, the width governs the maximum allowable $\Delta x$ and thereby the maximum force and its resolution that the sensor can measure.

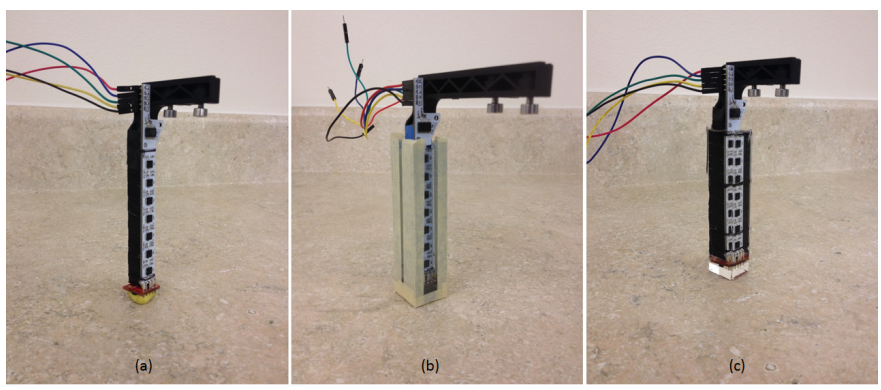

Fig. 3. (a) Sensors are mounted on the parallel gripper, (b) placed in an acrylic mold and (c) cured.

Once the object touches the sensor surface, i.e. $x=0$, (2) reduces to a constant, which is only a function of material properties. After touching, the PDMS gets compressed by $\Delta x \approx \frac{d F}{E A}$, leading to

$$
I_{x<0} \approx I_{0} \frac{\alpha}{\left(d-\frac{d F}{E A}\right)^{2}}-\kappa I_{0}
$$

Note that (4) still depends on the surface reflectance $\alpha$, which therefore needs to be known for accurate force measurements. we observe, however, that $x=F=0$ is an inflection point of the signal that can be potentially detected in the sensor's response and therefore allow to detect contact independently of surface properties. Indeed, equations (2) and (4) yield the same values for $x=0$ and $F=0$, respectively, but have different slopes. We experimentally show further below that this is the case and can be detected in the sensor readings.

\section{B. Manufacturing}

The infrared sensors requires few external components (3 capacitors). Encapsulation of the sensor in PDMS can be readily accomplished by mounting the sensors on a printed circuit board (PCB), placing it in a mold, and pouring the two-component liquid polymer in it (Figure 3). Painters tape has been used to ensure that the PDMS does not leak.

In order to avoid air being trapped at the interface between PDMS and the sensor, we degas the assembly in a vacuum chamber. The PDMS is then cured in an oven at $70^{\circ} \mathrm{C}$ for 20 minutes. This forms a robust and compliant rubber contact surface for grasping and manipulation. To improve repeatability of the optical properties of amorphous PDMS, it is advised to purify the raw materials before the mixing process to avoid extrinsic losses, e.g. by particle scattering. The base material and coupling agents should thus be filtered using a mixed cellulose ester membrane filter of pore size of $0.2 \mu \mathrm{m}$. The entire process takes around 5 hours to prepare a pair of these sensors. 


\section{Design PARAMETERS}

To experimentally characterize the performance of the proposed tactile sensor, we first characterized the response of an individual sensor and then the sensing capabilities of a complete array by installing it on a parallel gripper. Figure 4 shows the experimental setup to test and characterize the performance of these sensors. The setup is designed in a way which allows the testing of both, an individual sensor and complete arrays in their proximity and force regimes. The setup consists of a $0.15 \times 0.13 \mathrm{~m}^{2}$ screen that is mounted vertically on a sliding rod with a precise linear control. A digital force gauge (Shimpo FGV10XY) is mounted horizontally on the opposite side of the screen to measure the force exerted on the sensor.

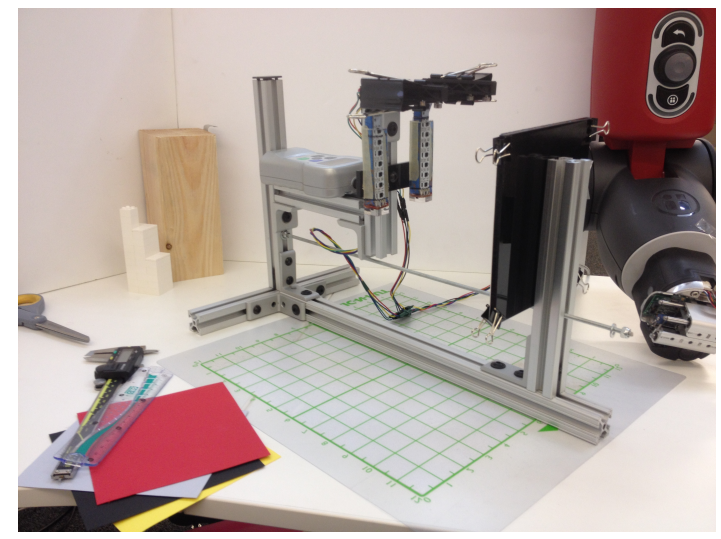

Fig. 4. Experimental setup to conduct proximity and force measurements.

Considering the air-PDMS surface as the screen's zero position, the screen is moved in discrete steps towards the sensor thereby measuring distance. After the screen touches the surface of the PDMS, the screen is rigidly fixed at a place where the force gauge reads $1 \mathrm{~N}$. A total of five readings are then taken at intervals of $1 \mathrm{~N}$ by further compressing the screen upon the sensor. Note that this approach allows us to seamlessly measure both distance and force as well as experimentally detect contact, which is defined by the force sensor changing from zero Newton to a positive value.

We have been using this setup to study the effects of various fabrication parameters, particularly the thickness of the PDMS film, mixing ratio of base to curing agent of pre-polymer and the operating sensor current value on the sensors response.

\section{A. Current}

We recorded single-point measurements at distances from 0 to $6 \mathrm{~cm}$ in increments of $1 \mathrm{~cm}$, as well as force from $0 \mathrm{~N}(0 \mathrm{~cm})$ to $5 \mathrm{~N}$ in increments of $1 \mathrm{~N}$ for current values from $40 \mathrm{~mA}$ to $200 \mathrm{~mA}$ in increments of $40 \mathrm{~mA}$ (Figure 5). PDMS with mixing ratio 8:1 has been applied to a thickness of $6 \mathrm{~mm}$ above the sensor. Results show saturation of the sensor for distances below $1 \mathrm{~cm}$ at current values exceeding $80 \mathrm{~mA}$ due to Fresnel reflection inside the
PDMS. At $80 \mathrm{~mA}$, the sensor saturates at less than $2 \mathrm{~N}$ force, whereas a $40 \mathrm{~mA}$ setting allows to measure across the range from 0 to $5 \mathrm{~N}$. Changing the current therefore allows trading range for resolution. At $80 \mathrm{~mA}$, distances from 0 to $6 \mathrm{~cm}$ result to ADC values from 5086 to 45789 or 40703 distinct values. At $40 \mathrm{~mA}$, the same range results to only 20245 values, corresponding to a resolution of $2.96 \mu \mathrm{m}$ and $1.47 \mu \mathrm{m}$. While not relevant given the accuracy of the sensor being four orders of magnitude worse than its resolution, in particular at longer distances, reducing the current allows extending the sensor's range from around $2 \mathrm{~N}$ to $5 \mathrm{~N}$ at an ADC resolution of $80 \mu \mathrm{N}$ and $285 \mu \mathrm{N}$, respectively.

\section{B. Thickness}

The thickness of PDMS has a considerable effect on the amount of light absorbed and scattered within the material. (The amount of light reflected back from the air-PDMS surface does not change as it depends only on the refractive indexes of the material.) Figure 5 middle, shows the response of two sensors cast in PDMS with the base to curing agent in 8:1 ratio and thickness of $6 \mathrm{~mm}$ and $12 \mathrm{~mm}$. Considering distance measurements from 0 to $6 \mathrm{~cm}$, absorption within the material compresses the range of raw ADC values from [5084;48201] for $6 \mathrm{~mm}$ PDMS coating to [3956;25612] for $12 \mathrm{~mm}$ PDMS coating, thereby reducing the resolution of the sensor for the 0 $6 \mathrm{~cm}$ range from $1.39 \mu \mathrm{m}$ to $2.77 \mu \mathrm{m}$. This effect is more accentuated in the force domain where the ADC values for measurements from 0 to $1 \mathrm{~N}$ range from 48454 to 64824 (16370 individual values or approximately $60 \mu \mathrm{N}$ resolution) for $6 \mathrm{~mm}$ PDMS coating, but span only a range of approximately 1000 values for the entire range of 0 to $5 \mathrm{~N}$ for $12 \mathrm{~mm}$ PDMS coating. For thicker coatings, we also observe a systematic non-monoticity of the signal for small forces, which is consistent for different current values and surface reflectance, and whose origin we currently cannot explain and which we wish to study in further work.

\section{Mixing Ratio}

The mid-infrared transmission of thin PDMS film is characterized in [9] using Fourier Transform Infrared Spectrometry. The transmittance of infrared light is found to depend strongly on the mixing ratio of base and curing agents causing the composition of PDMS to change; lower mixing ratios results in higher transmittance. Maximum transmittance of about $95 \%$ is found between wavenumbers $2490-2231 \mathrm{~cm}^{-1}$ with mixing ratios of $8: 1$. To compare the results at wavenumbers $12500-10526 \mathrm{~cm}^{-1}$ (800-950 $\mathrm{nm}$ ), we prepared three mixtures of PDMS with different mixing ratio of the base and curing agent (5:1, 10:1, and 12:1). Figure 5 shows the sensor proximity and force values for different mixing ratios. Albeit the Young's modulus of PDMS changes by about 35-40\% where the density changes by only $1 \%$ over the range of mixing ratio from $8: 1$ to $12: 1[1$, there is only little difference in the force region 

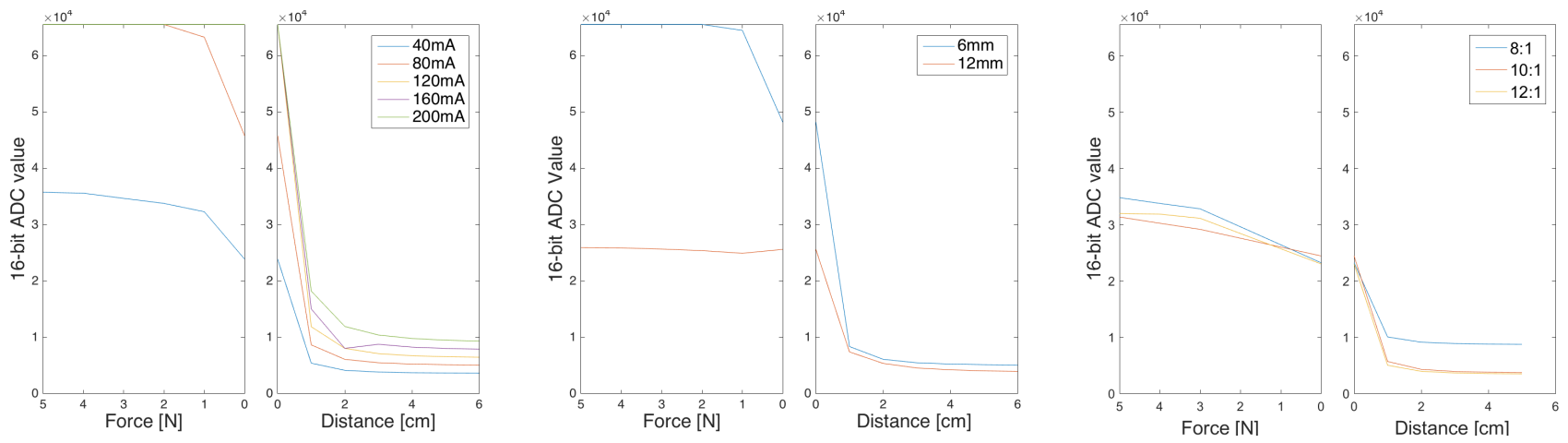

Fig. 5. Design parameters of the sensor. Left: Sensor response for different current settings. Middle: Sensor readings as a function of PDMS thickness at $80 \mathrm{~mA}$. Right: Sensor readings as a function of mixing ratio of the two PDMS components.

among these values, whereas distance measurements are more distinct, in particular for 8:1 mixing ratios. As the cross-over from distance to force is at approximately the same sensor reading, we deduct that 8:1 mixing ratios provide the widest dynamic range in the force regime, but the smallest dynamic range in the distance regime.

\section{iV. Calibration}

For calibrating the relationship between sensor reading and actual distance, we first characterize the sensitivity of the sensor to surface reflectance, and then record data for different distances across a variety of sensors for white paper. We have chosen a width of $6 \mathrm{~mm}$ at a mixing rate of $8: 1$ for the remainder of this paper due to the higher dynamic range in the distance and force regime, respectively.

\section{A. Color}

The intensity of light reflected from objects are greatly dependent on the color, pose and surface properties of the object. We chose five different colored target objects (red, yellow, white, gray and black, Canson, $150 \mathrm{gsm}$ ) as described in 3 . The colored cardboard papers were mounted on the screen shown in figure 4 which served as target objects for a distance sensor coated by $6 \mathrm{~mm}$ PDMS at 8:1 mixing ratio.

Figure 6 shows the response of the sensor to different colors. The proximity measurements are comparatively lesser influenced by the reflective properties of the target surface than the force measurements. Albeit bright colored materials give better readings than darker ones, there is not any significant difference in the sensor response to different colors, except for the black paper.

These findings are in line with [3], who calculate the reflectance for a variety of colors to be in the range of 0.9 (gray) to 1.0 (white), whereas black cardboard has a reflectance of 0.12 . Cardboard of all colors is much more reflecting than wood (0.77), brick (0.61) or concrete (0.53), but much less than reflecting surfaces such as polished plastic or china.

\section{B. Distance}

In order to obtain a relationship between sensor readings and actual distance we recorded data from 14 different sensors and white paper. We soldered seven sensors in a line at $10 \mathrm{~mm}$ spacing to a rigid PCB (Figure 1). We recorded the response of two such arrays (14 sensors) at 24 distances ranging from 0.5 to $19 \mathrm{~cm}$ and 50 measurements each for $120 \mathrm{~mA}$. Albeit $120 \mathrm{~mA}$ leads to saturation in the force regime (when using white paper), this value allows us to obtain better ranging and works fine with objects that are less reflecting. The data is shown in Figure 6

We fitted this data with a function of the form $y=$ $a x^{b}+c$ using MATLAB's curve fitting toolbox's trustregion method and bisquare weighting of outliers. The candidate function corresponds to physical intuition (with $b=-2$ ) and can be inverted to

$$
x=\frac{1}{((y-c) / a)^{\frac{1}{b}}}
$$

Notice that the denominator of the above equation includes the b-th root, which yields complex values for $y<c$. This happens whenever a sensor reading falls below the assymptote of the fitted curve, which is often the case for farther-away measurements. We therefore convert all measurements into decibel scale using $\log _{10} \frac{I}{I_{\infty}}$, where $I_{\infty}$ is the measurement obtained in plein air. With $b \approx-1$ after fitting on the log-scale, all distance measurements remain real. The fit as well as absolute error for both the raw and PDMS-coated sensors are shown in Figure 6 . middle. As expected, we observe a slightly higher absolute error for all measurements with PDMS, which initially makes objects appear closer (up to about $7 \mathrm{~cm}$ ) and then farther apart than the raw sensor. Data follows a similar trend for distances from $10 \mathrm{~cm}$ to $19 \mathrm{~cm}$, but are not shown as the high error at this range makes those measurements impractical to use.

\section{Force}

As force measurements are much more susceptive to surface reflectance, we have performed fits for a variety of 

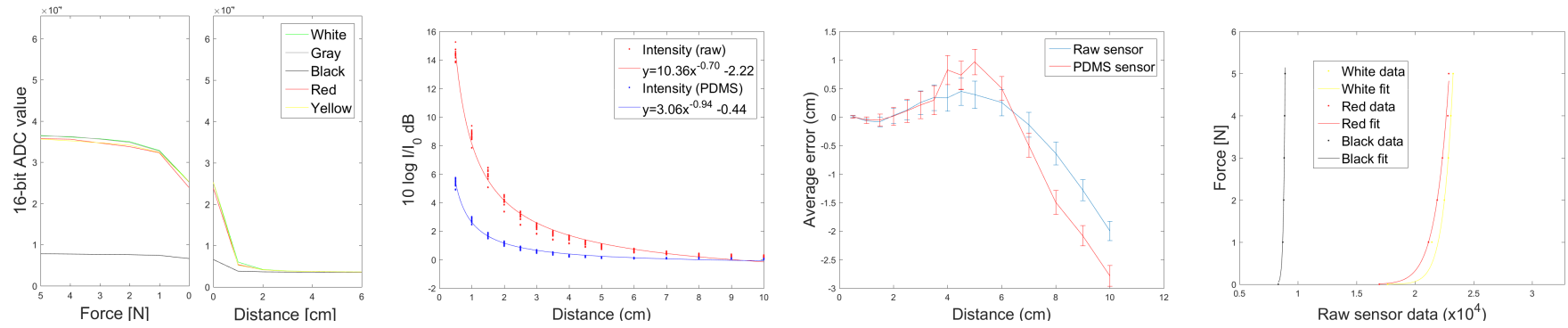

Fig. 6. Left: Sensor response (PDMS 6mm, 8:1 mixing ratio) to different color targets. Middle two: Sensor response to different distances at $120 \mathrm{~mA}, 6 \mathrm{~mm}$ PDMS with 8:1 mixing ratio averaged over 14 different sensors. Average error after applying calibration to raw data. Right: Raw sensor readings vs. actual force for different colors and fit of the form $y=a x^{b}$.

colored papers using data from Figure 6, left. Results for a subset (white, red, black) are shown in Figure 6 , right. Using an equation of the form $y=a x^{b}$ has provided good results, with R-squared values ranging from 0.9898 (black) to 0.9953 (white).

\section{Applications}

We mount our finger sensors on the parallel gripper of the Baxter robot from Rethink Robotics, which is equipped with two 7-DOF arms. The size of each finger sensor is $80 \times 2 \times 1 \mathrm{~mm}$ (Figure 1), small enough to install on the stock electric parallel gripper of the robot. We manufactured two separate pairs of finger set with grasp ranges varying from $0-68 \mathrm{~mm}$ to $68-144 \mathrm{~mm}$. Two fingers are interfaced via an Arduino Uno microcontroller that polls all 16 sensors in a round-robin fashion. The microcontroller connects to a control computer and ROS via an USB port, which also provides the supply voltage for the sensors. Unless otherwise noted, all objects are chosen from the Yale-CMU-Berkeley (YCB) Object and Model set [8].

\section{A. Gripper centering}

We first investigate using proximity sensing to center a gripper around an object. This is important when successful grasping requires both fingers to simultaneously make contact. For example grasping a cup at its handle induces a turning motion that needs to be counteracted by the opposite finger before the cup has turned out of the robot's grasp. Similarly, removing a block from a Jenga tower 25] requires to create force-closure with the block while inducing a minimum amount of motion on the block itself.

Figure 7 depicts a similar situation, in which imprecise alignment will collapse a tower of wooden blocks. The grippers were closed in discrete steps and the response form the sensor was recorded. The response from the sensors on the right finger is shown in blue and the response from the left finger is shown in red.

Assuming the surface properties (reflectance) are the same on both sides of the object, data shown in Figure 7 can be used to servo the end-effector to a position, in which both distances are roughly equal using simple feedback
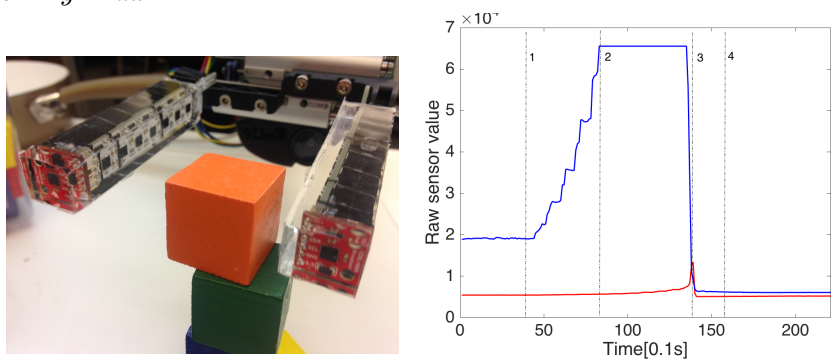

Fig. 7. Deconstructing a tower of 1-inch cubes requires exact centering of the gripper in order to prevent the cubes from falling. The plot to the right shows raw sensing values for the left (blue) and right (red) finger. The left gripper approaches the cube in phase 1-2. It makes contact at 2 while the right finger continues its approach during $2-3$. As the left gripper is still pushing the cube, it will eventually fall of the green cube and disappears from both grippers' field of view $(3-4)$.

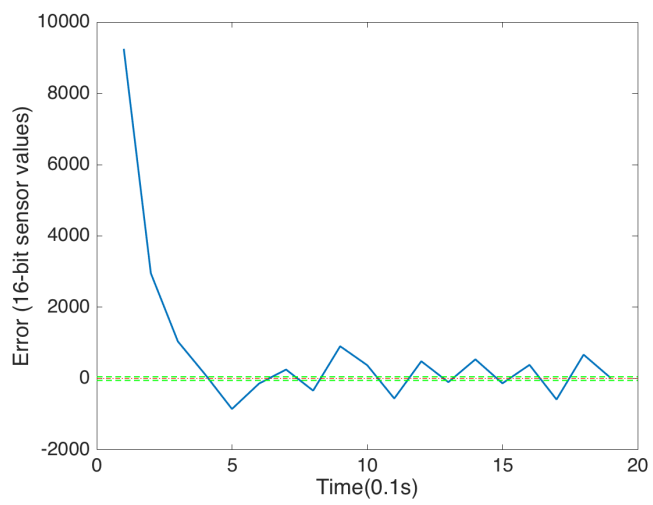

Fig. 8. Using the average of differences in sensor readings measured from the left and right finger (Figure 7) to center an object.

control and inverse kinematics (Baxter SDK PyKDL), see Figure 8

\section{B. Contact point evaluation}

Force sensing can be used to determine the location of incidence of an object on the gripper. Figure 9 left, shows the raw measurements of all sensors when grasping the handle of the YCB pan (Figure 10 left). The data clearly shows the fingers approaching the handle and which sensors made the most contact, letting us infer the approximate size of the object. Closer inspection of 

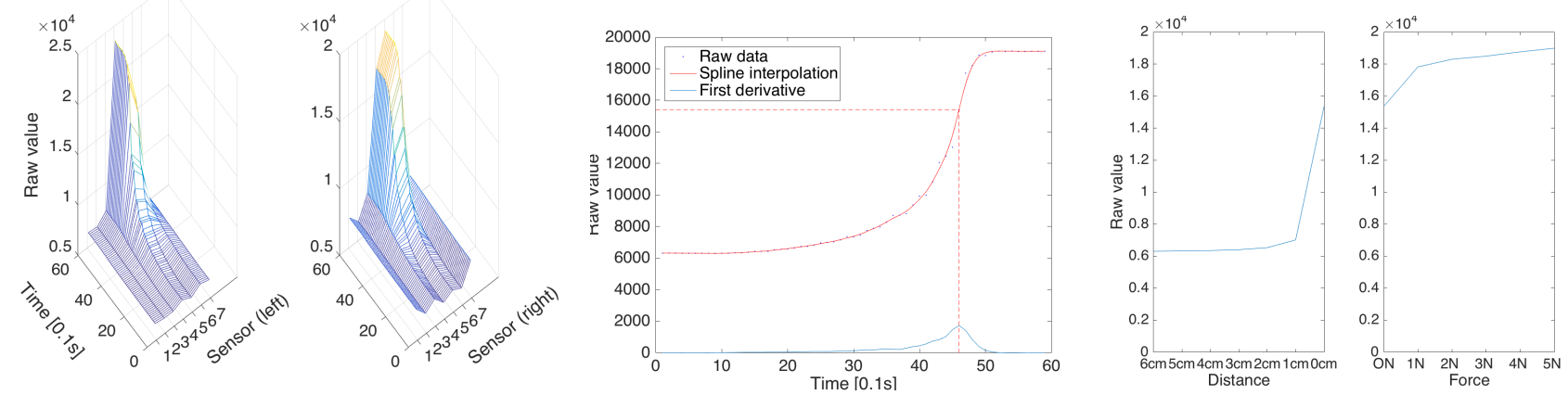

Fig. 9. Left: Raw sensor values on the left and right finger when grasping the YCB pan at its handle during roughly $6 \mathrm{~s}$. The contact points on each finger are clearly discernible. Middle: Close-up on sensor 5 of the right finger and its numerical derivative. Horizontal and vertical lines indicate the raw value that corresponds to the derivative's peak. Right: Calibration data for black cardboard. Horizontal and vertical lines indicate the raw value that corresponds to crossing from distance to force.

contact data, here the 5 th sensor of the right finger, reveals that gentle pressure drives the sensor to roughly $2 \times 10^{4}$ (Figure 10 middle), which is similar to values generated by contact with black card-board (Figure 10 , right). Furthermore, fitting a spline to the raw data and calculating its derivative (MATLAB spline and fnder), reveals that the sensor response has an inflection point close to where the black cardboard crosses from the distance to the force regime. Performing the same operation on data from the left finger suggests a material of slightly higher reflectance (the sensor maxes out at $2.4 \times 10^{4}$ ) with a cross-over point at a raw value of 17529 . Albeit not sufficient to determine the actual object properties, we wish to explore the suitability of using extrema on the sensor response (minimum, maximum, and cross-over point) to identify specific materials in the future. Indeed, we are able to correctly detect the cross over point for all experiments shown in Figure 6 (five different colors), left, for currents ranging from $40 \mathrm{~mA}$ to $200 \mathrm{~mA}$ in increments of $40 \mathrm{~mA}$ (25 experiments).

\section{3D Reconstruction}

Given the material parameters, in-hand proximity sensing can be used to augment, and possibly register against, conventional $3 \mathrm{D}$ sensing. The robot arm is programmed
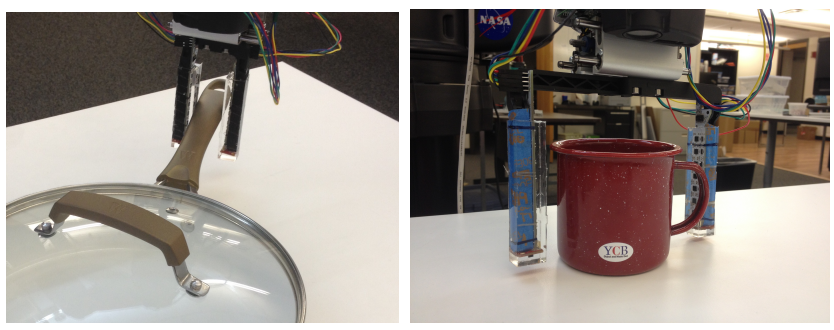

Fig. 10. Left: Grasping the YCB pan. Right: Scanning the YCB cup by performing a 360 degree swivel.

to reach a specified scanning position on the table as shown in Figure 10 We assume such a position can be reached using coarse visual or RGB-D data, as well as the proximity sensors themselves. The robot wrist joint is rotated around the object in increments of $0.17 \mathrm{rad}$ in the interval of $[-\pi ; \pi]$. Using the actual encoder value at each step and converting sensor readings into centimeters using (5) yields polar coordinates of each point where the infrared light hits the object. The resulting data is shown in Figure 11 Albeit noisy due to non-orthogonal incidence angles at the handle and the bottom of the cup, the fidelity of the model is sufficient to highlight the presence of the cup's handle and is, after removing outliers, close $( \pm 0.5 \mathrm{~cm})$ to the cup's true diameter.
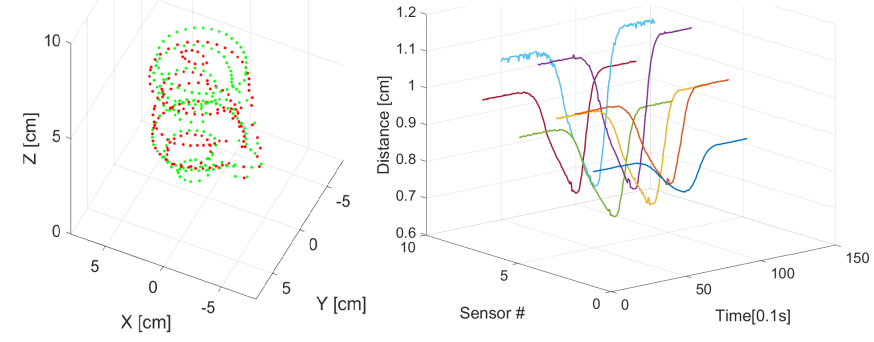

Fig. 11. Left: 3D point cloud model of a cup $(8 \mathrm{~cm}$ diameter, $8 \mathrm{~cm}$ height, $3 \mathrm{~cm}$ handle). The red colored points are from the left finger sensor and the green colored points from the right finger sensor. Right: Horizontal sweep across an airplane wing, only data from the right finger is shown.

We also selected a toy airplane form the YCB object set which has a highly reflecting surface. Figure 12 shows the direction in which the robot wrist is swept across the wing to detect a possible grasp location, this time using a horizontal motion.

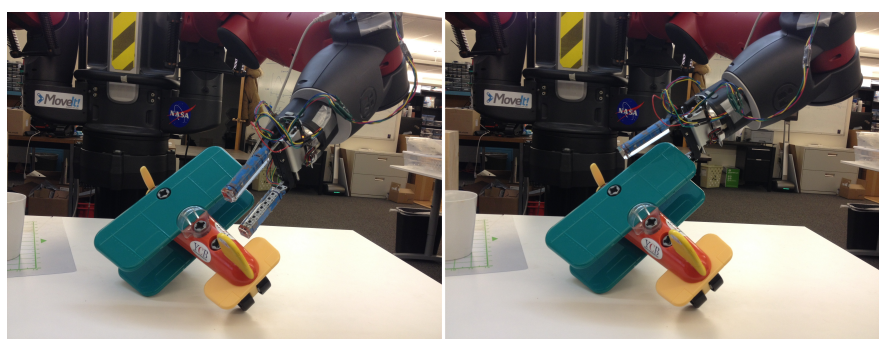

Fig. 12. Scanning a toy airplane wing. 
Figure 11] right, shows the response of the sensors. Albeit the distance measurements are underestimated due to the reflectance of the opposite gripper (actually at a distance of around $4 \mathrm{~cm}$ ) and the airplane wing, the presence of the airplane wing is clearly discernible. As the wing starts appearing in the field of view of the sensors we see a steep decrease in distance, which then reaches a minimum at the wing's center, and then gradually increases as the robot arm moves away from the wing. This is due to the fact that the infrared emitter is better approximated by a lobe than by a ray. The symmetry of the reflected plane at the center of the wing causes the photo receiver to receive the maximum possible reflected intensity available from the airplane wing, illustrating the limitations in lateral resolution, which would need to be compensated by an orthogonal sweep, should a more accurate $3 \mathrm{D}$ reconstruction be desired.

\section{Discussion}

The proposed sensor has a series of design parameters, ranging from the choice of the material itself, its mixing ratio, its thickness, and the current at which the emitter operates. Each of these parameters affects the sensors' range, dynamic range, and thereby resolution and accuracy. While far from exhaustive, systematic experiments presented here highlight important trends, and allow to obtain a good trade-off between ranging and force sensing capabilities, as well as providing guidelines on how to adapt the sensor to one's own specifications.

Albeit roughly following the form $y=a x^{b}+c$, our approximation introduces non-negligible systematic error, an effect that gets amplified by adding a PDMS layer, which introduces another constant to the denominator of (2). While better non-linear approximations could be found, e.g., using support vector machines or training a neural network, the sensor is very sensitive to surface properties. For example, black paper is five times less reflecting than white paper, whereas shiny objects are more reflecting than objects with rougher surfaces. We observe, however, that most practical application of the proposed sensor might not require calibration at all. Indeed, centering around an object only requires equalizing raw sensor readings, which are both monotonically increasing and continuous from infinite distance to at least $5 \mathrm{~N}$ force.

We also observe that the shape of the function that relates distance/force measurements to raw sensor readings is of similar quality independent of the surface properties, thickness, mixing ratio, and current, with an inflection point at the contact point. Performing a firm grasp on an unknown object such as the panhandle in Figure 9 allows to record such a curve in its entirety and might allow to infer its material properties given all other parameters of the sensor are known. For example, when squeezing the handle, the sensor reading maxes out at around $2.1 \times 10^{4}$, which is slightly above the value of black paper at $120 \mathrm{~mA}$ $\left(1.8 \times 10^{4}\right)$ for $6 \mathrm{~mm}$ PDMS (8:1). Together with actual distance information obtained from the gripper itself, it might be possible to calibrate the sensor online by performing a simple grasp, and then use this data to perform an accurate 3D reconstruction. Squeezing an object might also provide insight for tuning the sensing current. For example, the sensor current could be reduced until the sensor saturates at a value below the maximum reading, and calibration data could be obtained during a second squeeze. Finally, tactile and geometry information could complement existing approaches in which robots use active manipulation to understand objects in their environment [33, 28.

Another limitation of optical proximity sensors is their dependence on the angle of incidence. While this is not noticed with rotation symmetric objects such as those used here, scanning a rectangular object using a circular swivel motion, e.g., would let the object appear elliptically. As the resulting error is well quantified [3], we wish to investigate how contact information can be exploited to estimate the angle of a surface, and investigate sensorbased motion planning techniques that can help the robot to differentiate between accurate and erroneous signals by automatically testing different hypothesis of the true object's pose and surface properties.

\section{CONCLUSION AND FURTHER WORK}

We present an integrated force and distance sensor that is simple to manufacture and low-cost, yet provides a series of benefits that usually require much more complex sensors. We experimentally evaluate the design space and demonstrate the impact of different material properties and control parameters on the sensor response. As expected with infrared-based sensors, the sensor is strongly non-linear, highly sensitive to surface properties and has poor lateral resolution when compared with ray-based or RGB-D sensors. Nevertheless, we demonstrate that the proposed sensor has a wide range of use cases that facilitate grasping and manipulation ranging from contact point detection, determining grasp points, to object registration, and can possibly be improved by better sensor models and sensor-based motion planning strategies, which we wish to investigate in the future. We are also interested to explore real-time object recognition by squeezing and scanning an object. In the long run, the necessary processing could be co-located with the sensor [30, allowing it to autonomously identify surface properties of an object and adapting accordingly.

\section{ACKNOWLEDGMENTS}

The authors would like to thank Michael Bernabei for initial testing, Dana Hughes and John Klingner for PCB design, Jorge Canardo, Nick Farrow, Chris Heckmann, and Andy McEvoy for helpful discussion, and Keyon Janani and Carrie Weidner with help on sensor characterization. This work has been supported by AFOSR grant FA955015-1-0238. We are grateful for this support. 


\section{REFERENCES}

[1] Deniz Armani, Chang Liu, and Narayan Aluru. Reconfigurable fluid circuits by PDMS elastomer micromachining. In Micro Electro Mechanical Systems, 1999. MEMS'99. Twelfth IEEE International Conference on, pages 222-227. IEEE, 1999.

[2] DJ Balek and RB Kelley. Using gripper mounted infrared proximity sensors for robot feedback control. In Robotics and Automation. Proceedings. 1985 IEEE International Conference on, volume 2, pages 282287. IEEE, 1985.

[3] Gines Benet, Francisco Blanes, José E Simó, and Pascual Pérez. Using infrared sensors for distance measurement in mobile robots. Robotics and Autonomous systems, 40(4):255-266, 2002.

[4] Jeannette Bohg, Aythami Morales, Tamim Asfour, and Danica Kragic. Data-driven grasp synthesis - a survey. Robotics, IEEE Transactions on, 30(2):289309, 2014.

[5] Max Born and Emil Wolf. Principles of optics: electromagnetic theory of propagation, interference and diffraction of light. Cambridge University press, 1999.

[6] Dengke Cai, Andreas Neyer, Rüdiger Kuckuk, and H Michael Heise. Raman, mid-infrared, near-infrared and ultraviolet-visible spectroscopy of pdms silicone rubber for characterization of polymer optical waveguide materials. Journal of Molecular Structure, 976 (1):274-281, 2010.

[7] Ziliang Cai, Weiping Qiu, Guocheng Shao, and Wanjun Wang. A new fabrication method for all-PDMS waveguides. Sensors and Actuators A: Physical, 204: 44-47, 2013.

[8] Berk Calli, Aaron Walsman, Arjun Singh, Siddhartha Srinivasa, Pieter Abbeel, and Aaron M Dollar. Benchmarking in manipulation research: The YCB object and model set and benchmarking protocols. arXiv preprint arXiv:1502.03143, 2015.

[9] KC Chen, AM Wo, and YF Chen. Transmission spectrum of PDMS in 4-7 $\mu \mathrm{m}$ mid-IR range for characterization of protein structure. In NSTI-Nanotech, volume 2, pages 732-735, 2006.

[10] Nikolaus Correll, Kostas E. Bekris, Dmitry Berenson, Oliver Brock, Albert Causo, Kris Hauser, Kei Okada, Alberto Rodriguez, Joseph M. Romano, and Peter R. Wurman. Lessons from the Amazon picking challenge. arXiv:1601.05484, 2016.

[11] Nikhil Chavan Dafle, Alex Rodriguez, Robert Paolini, Bowei Tang, Siddhartha S Srinivasa, Michael Erdmann, Matthew T Mason, Ivan Lundberg, Harald Staab, and Thomas Fuhlbrigge. Extrinsic dexterity: In-hand manipulation with external forces. In Robotics and Automation (ICRA), 2014 IEEE International Conference on, pages 1578-1585. IEEE, 2014.

[12] Ravinder S Dahiya, Giorgio Metta, Maurizio Valle, and Giulio Sandini. Tactile sensing: from humans to humanoids. Robotics, IEEE Transactions on, 26(1): 1-20, 2010.

[13] Ravinder S Dahiya, Philipp Mittendorfer, Maurizio Valle, Gordon Cheng, and Vladimir J Lumelsky. Directions toward effective utilization of tactile skin: A review. Sensors Journal, IEEE, 13(11):4121-4138, 2013.

[14] Raphael Deimel and Oliver Brock. A novel type of compliant, underactuated robotic hand for dexterous grasping. Robotics: Science and Systems, Berkeley, $C A$, pages 1687-1692, 2014.

[15] Aaron M Dollar, Leif P Jentoft, Jason H Gao, and Robert D Howe. Contact sensing and grasping performance of compliant hands. Autonomous Robots, 28 (1):65-75, 2010.

[16] Nicholas Farrow and Nikolaus Correll. A soft pneumatic actuator that can sense grasp and touch. In $I n-$ telligent Robots and Systems (IROS), 2015 IEEE/RSJ International Conference on, pages 2317-2323. IEEE, 2015.

[17] Nicholas Farrow, Yang Li, and Nikolaus Correll. Morphological and embedded computation in a selfcontained soft robotic hand. arxiv:1605.00354, 2016.

[18] Yuki Fujimori, Yoshiyuki Ohmura, Tatsuya Harada, and Yasuo Kuniyoshi. Wearable motion capture suit with full-body tactile sensors. In Robotics and $A u$ tomation, 2009. ICRA'09. IEEE International Conference on, pages 3186-3193. IEEE, 2009.

[19] D Goger, Hosam Alagi, and Heinz Wörn. Tactile proximity sensors for robotic applications. In Industrial Technology (ICIT), 2013 IEEE International Conference on, pages 978-983. IEEE, 2013.

[20] Greg Hellard and R Andrew Russell. A robust, sensitive and economical tactile sensor for a robotic manipulator. In Australian Conference on Robotics and Automation, pages 100-104. Citeseer, 2002.

[21] Kaijen Hsiao, Paul Nangeroni, Manfred Huber, Ashutosh Saxena, and Andrew Y Ng. Reactive grasping using optical proximity sensors. In Robotics and Automation, 2009. ICRA'09. IEEE International Conference on, pages 2098-2105. IEEE, 2009.

[22] Dana Hughes and Nikolaus Correll. Texture recognition and localization in amorphous robotic skin. Bioinspiration \& biomimetics, 10(5):055002, 2015.

[23] Leo Jiang, Kevin Low, Joey Costa, Richard J Black, and Yong-Lae Park. Fiber optically sensorized multifingered robotic hand. In Intelligent Robots and Systems (IROS), 2015 IEEE/RSJ International Conference on, pages 1763-1768. IEEE, 2015.

[24] Jelizaveta Konstantinova, Agostino Stilli, and Kaspar Althoefer. Force and proximity fingertip sensor to enhance grasping perception. In Intelligent Robots and Systems (IROS), 2015 IEEE/RSJ International Conference on, pages 2118-2123. IEEE, 2015.

[25] Torsten Kröger, Bernd Finkemeyer, Simon Winkel- 
bach, Lars-Oliver Eble, Sven Molkenstruck, and Friedrich M Wahl. A manipulator plays Jenga. Robotics $\&$ Automation Magazine, IEEE, 15(3):7984, 2008.

[26] S.S. Lancaster. A fuzzy logic controller for the application of skin pressure. In Fuzzy Information, 2004. Processing NAFIPS '04. IEEE Annual Meeting of the, volume 2, pages 686-689 Vol.2, June 2004. doi: 10.1109/NAFIPS.2004.1337384.

[27] Hyung-Kew Lee, Jaehoon Chung, Sun-Il Chang, and Euisik Yoon. Normal and shear force measurement using a flexible polymer tactile sensor with embedded multiple capacitors. Microelectromechanical Systems, Journal of, 17(4):934-942, 2008.

[28] Lu Ma, Mahsa Ghafarianzadeh, Dave Coleman, Nikolaus Correll, and Gabe Sibley. Simultaneous localization, mapping, and manipulation for unsupervised object discovery. In IEEE International Conference on Robotics and Automation, pages 1344-1351, 2015.

[29] Alexis Maldonado, Humberto Alvarez, and Michael Beetz. Improving robot manipulation through fingertip perception. In Intelligent Robots and Systems (IROS), 2012 IEEE/RSJ International Conference on, pages 2947-2954. IEEE, 2012.

[30] MA McEvoy and N Correll. Materials that couple sensing, actuation, computation, and communication. Science, 347(6228):1261689, 2015.

[31] P Mittendorfer, E Yoshida, and G Cheng. Realizing whole-body tactile interactions with a self-organizing, multi-modal artificial skin on a humanoid robot. $A d$ vanced Robotics, 29(1):51-67, 2015.

[32] Philipp Mittendorfer and Gordon Cheng. Humanoid multimodal tactile-sensing modules. Robotics, IEEE Transactions on, 27(3):401-410, 2011.

[33] John Oberlin and Stefanie Tellex. Learning to pick up objects through active exploration. In Development and Learning and Epigenetic Robotics (ICDLEpiRob), 2015 Joint IEEE International Conference on, pages 252-253. IEEE, 2015.

[34] Jonathan Rossiter and Toshiharu Mukai. An ledbased tactile sensor for multi-sensing over large areas. In Sensors, 2006. 5th IEEE Conference on, pages 835838. IEEE, 2006.

[35] Yaroslav Tenzer, Leif P Jentoft, and Robert D Howe. The feel of mems barometers: inexpensive and easily customized tactile array sensors. Robotics \& Automation Magazine, IEEE, 21(3):89-95, 2014.

[36] Lin Wang and David J Beebe. Characterization of a silicon-based shear-force sensor on human subjects. Biomedical Engineering, IEEE Transactions on, 49 (11):1340-1347, 2002.

[37] Zhi Feng Zhang, Xiao Ming Tao, Hua Peng Zhang, and Benpeng Zhu. Soft fiber optic sensors for precision measurement of shear stress and pressure. Sensors Journal, IEEE, 13(5):1478-1482, 2013.

[38] F Zhu and JW Spronck. A capacitive tactile sensor for shear and normal force measurements. Sensors and Actuators A: Physical, 31(1):115-120, 1992. 